\title{
The Relationship between Personality, Sense of Efficacy, and Stress in Korean Teachers
}

\author{
Subin Park', Yul-Mai Song², Guy-Nueo Ko², Kyungun Jhung ${ }^{3}$, \\ Kyooseob $\mathrm{Ha}^{4,5}$, Young-Ryeol $\mathrm{Lee}^{6}$, and Yeni Kim${ }^{2}$ \\ ${ }^{1}$ Department of Research Planning, Mental Health Research Institute, National Center for Mental Health, Seoul, Republic of Korea \\ ${ }^{2}$ Department of Child and Adolescent Psychiatry, National Center for Mental Health, Seoul, Republic of Korea \\ ${ }^{3}$ Department of Psychiatry and Behavioral Neurosciences, International St. Mary's Hospital, Catholic Kwandong University, Incheon, Republic of \\ Korea \\ ${ }^{4}$ Department of Psychiatry, National Center for Mental Health, Seoul, Republic of Korea \\ ${ }^{5}$ Department of Psychiatry and Behavioral Science, Seoul National University College of Medicine, Seoul, Republic of Korea \\ ${ }^{6}$ Department of Psychiatry, Bugok National Hospital, Changyeong, Republic of Korea
}

\begin{abstract}
Several studies have linked teachers' personality characteristics and sense of efficacy to stress. However, investigating the relationship between these three constructs in this context was limited. This study aims to investigate the relationship between personality, sense of efficacy and perceived stress among Korean teachers. A total of 137 teachers working in elementary, middle, and high schools located in Seoul, South Korea were recruited for the study. The participants were administered Temperament and Character Inventory, Teacher's Sense of Efficacy Scale (TSES), and Perceived Stress Scale. The TSES was negatively correlated with harm avoidance and positively correlated with persistence, self-directedness, cooperativeness, and self-transcendence. Perceived stress was positively correlated with harm avoidance and negatively correlated with persistence and self-directedness. The path analysis showed that harm avoidance directly predicted perceived stress $(\beta=0.37,95 \% \mathrm{CI}=0.21-0.53, \mathrm{p}=0.002)$, and self-directedness and persistence predicted one's sense of efficacy $(\beta=0.18,95 \% \mathrm{CI}=0.01-0.39$ and $\beta=0.31,95 \% \mathrm{CI}=0.10-0.47)$, which predicted perceived stress $(\beta=-0.21,95 \% \mathrm{CI}=-0.39$ to -0.02$)$. The results of the present study indicate that harm avoidance might be associated with stress-proneness, while persistence, self-directedness, and sense of efficacy might act as protective resources against stress in Korean teachers.

Psychiatry Investig 2016;13(5):566-570
\end{abstract}

Key Words Personality, Stress, Self-efficacy, Teacher.

\section{INTRODUCTION}

Teachers play an important role in creating a classroom climate that fosters students' learning and social-emotional wellbeing. However, teaching can be stressful. Multiple sources of stress, including time demands, workload, disruptive student behavior, and organizational factors, have been cited. ${ }^{1,2}$ Teachers' stress can impact their teaching effectiveness and the quality of their interactions with students. In addition, teachers' perceptions of stress can compromise their wellbeing and lead to burnout. ${ }^{3}$ Two important psychological ele-

Received: September 24, 2015 Revised: January 12, 2016

Accepted: February 5, 2016 Available online: July 11, 2016

$\triangle$ Correspondence: Yeni Kim, MD, PhD

Department of Child and Adolescent Psychiatry, National Center for Mental Health, 398 Neungdong-ro, Gwangjin-gu, Seoul 04933, Republic of Korea Tel: +82-2-2204-0301, Fax: +82-2-2204-0356, E-mail: yeni1004@gmail.com

(a) This is an Open Access article distributed under the terms of the Creative Commons Attribution Non-Commercial License (http://creativecommons.org/licenses/by$\mathrm{nc} / 3.0$ ) which permits unrestricted non-commercial use, distribution, and reproduction in any medium, provided the original work is properly cited. ments-one's personality ${ }^{4,5}$ and sense of self-efficacy ${ }^{6-8}$ have been linked to greater teaching engagement and less emotional exhaustion and psychological distress.

An individual's personality can affect his or her stress appraisal and stress-coping mechanisms, but it is also crucial with regard to the selection and shaping of stressful situations. ${ }^{9}$ Stable personality characteristics predispose individuals to view stressful events in a certain way that can either impair or facilitate the adaptation process. ${ }^{10}$ Two major personality theories are the Big Five model, which uses the NEO-Personality Inventory (NEO-PI) as a measuring instrument, ${ }^{11}$ and the psychobiological theory of personality, which uses the Temperament and Character Inventory (TCI) as a measuring instrument. ${ }^{12}$ Several studies have investigated the relationship between the Big Five personality dimensions, measured by the NEO-PI, and stress. Besser and Shackelford ${ }^{13}$ have shown that higher conscientiousness is related to lower perceived stress. Modeling the stress process, Conard and Matthews ${ }^{14}$ have suggested that the primary driver of perceived stress is 
neuroticism. In occupational studies, personality variables, such as neuroticism, extraversion, and conscientiousness, have been identified as predictors of stress in teachers. ${ }^{4,5,15}$ However, no study has been found that analyzes the relationship between psychobiological theory of personality and perceived stress.

Self-efficacy is an individual's belief in his or her ability to organize and execute the courses of action required to produce particular outcomes. ${ }^{16}$ In the education field, a teacher's sense of efficacy refers to the beliefs that the teacher holds regarding his or her capacity to achieve desired instructional outcomes. ${ }^{17}$ Self-efficacy determines the cognitive appraisal of stressful situations, ${ }^{16}$ and both epidemiological and laboratory studies have shown that highly self-efficacious individuals perceive stressful situations as more challenging than stressful. ${ }^{18,19}$ Self-efficacy reportedly plays an important role in decreasing stress levels among teachers. ${ }^{6-8}$

According to transactional theory of stress and coping, ${ }^{20}$ an individual's reaction to the stress is mediated by the person's appraisal of the environment and the process of coping with a stressful event. Appraisals of the stressor and coping behavior are influenced by personal characteristics such as personality, perceived control, and sense of efficacy. Based on this theory, we considered not only the possibility that personality predicts perceived stress, but also the possibility that the effect of personality on the perceived stress is mediated through the effect of personality on sense of efficacy.

Although both the association between personality and perceived stress and between teachers' sense of efficacy and perceived stress have been confirmed in previous studies, the role of teachers' sense of efficacy as a mediator between their personalities and perceived stress has not been examined. This study thus examines the psychobiological theory of personality in terms of its interrelations with teachers' sense of efficacy and perceived stress. In addition to the mere correlations among the variables, this study will examine whether a mediation effect (personality $\rightarrow$ sense of efficacy $\rightarrow$ perceived stress) exists to gain more insight into possible mechanisms in the development of teachers' stress and protective factors. The study is guided by the assumption that personality constitutes a resource factor and that sense of efficacy may operate as mediator between psychobiological theory of personality and perceived stress.

\section{METHODS}

\section{Participants}

A total of 190 teachers working in elementary $(n=67)$, middle $(\mathrm{n}=38)$, and high $(\mathrm{n}=22)$ schools located in Seoul, South Korea, who participated in a camp that aimed to assess the mental health of teachers, were recruited for the study. The participants volunteered. Three tools were used for data collection: the TCI, the Teachers' Sense of Self-Efficacy Scale (TSES), and the Perceived Stress Scale (PSS). Before these tools were distributed to the participants for completion, the researchers explained the objectives of the study to the participants to ensure reliable data. The study was approved by the institutional review board (IRB) for human subjects at the Seoul National Hospital. Participants were included in the analyses for this study only if they completed all items of the TCI, the TSES, and the PSS. Fifty three study subjects were excluded because of missing data, leaving a total of 137 subjects ( 25 males, 112 females) in the analysis.

\section{Measurements}

\section{The Temperament and Character Inventory}

The TCI consists of 240 true-or-false questions, ${ }^{12}$ and validity and reliability studies of the Korean version have been performed..$^{21}$ Cloninger's model examines the temperament dimension by 4 scales: novelty seeking, harm avoidance, reward dependence, and persistence. It also examines the character dimension by 3 scales: self-directedness, cooperativeness, and self-transcendence.

\section{The Teachers' Sense of Efficacy Scale}

The TSES $^{22}$ was translated into Korean and adapted for this context. ${ }^{23}$ This scale consists of three dimensions: instructional strategies (e.g., How much can you do to adjust your lessons to the proper level for individual students?), classroom management (e.g., How much can you do to control disruptive behavior in the classroom?), and student engagement (e.g., How much can you do to get students to believe they can do well in schoolwork?). The Cronbach's alpha reliabilities for the subscales were 0.85 for instructional strategies, 0.75 for classroom management, and 0.86 for student engagement. The 9-point scale in the original version of the TSES was converted into a 5 -point Likert-type scale in this study ( $1=$ not at all; $5=$ a great deal).

\section{The Perceived Stress Scale}

The PSS was designed to measure the degree to which situations in one's life are appraised as stressful. ${ }^{24}$ The 5-point Likert-type scale consists of 10 items that assess the respondents' perceptions of stressful experiences by asking them to rate the frequency of their feelings and thoughts related to events and situations that have occurred over the previous month. Validity and reliability studies of the Korean version have been performed. ${ }^{25}$ 


\section{Statistical analysis}

Pearson correlation analysis was conducted to assess the inter-relationships among continuous variables using SPSS (version 21.0; SPSS Inc., Chicago, IL, USA). We used the AMOS (version 19.0; SPSS Inc., Chicago, IL, USA) to conduct path analyses. The TCI subscale scores-which were significantly correlated with the PSS scores-the TSS scores,

Table 1. Participants' characteristics

\begin{tabular}{lc}
\hline \multicolumn{1}{c}{ Characteristics } & $\mathrm{N}(\%)$ or mean (SD) \\
\hline Gender, females, N (\%) & $112(81.8)$ \\
Age, N (\%) & $4(2.9)$ \\
$20 \mathrm{~s}$ & $14(10.2)$ \\
$30 \mathrm{~s}$ & $31(22.6)$ \\
$40 \mathrm{~s}$ & $76(55.5)$ \\
$50 \mathrm{~s}$ & $12(8.8)$ \\
60s & \\
TCI, mean (SD) & $30.90(11.03)$ \\
Novelty seeking & $34.78(10.87)$ \\
Harm avoidance & $44.39(8.75)$ \\
Reward dependence & $44.13(10.36)$ \\
Persistence & $50.44(10.14)$ \\
Self-directedness & $57.48(9.40)$ \\
Cooperativeness & $34.43(12.02)$ \\
Self-transcendence & $52.37(7.63)$ \\
TSES, mean (SD) & $18.73(3.20)$ \\
Instructional strategies & $14.71(2.46)$ \\
Classroom management & $18.94(2.80)$ \\
Student engagement & $16.30(5.97)$ \\
PSS, mean (SD) & \\
\hline
\end{tabular}

TCI: Temperament and Character Inventory, TSES: Teachers' Sense of Efficacy Scale, PSS: Perceived Stress Scale and the PSS scores were included in the path model. Statistical significance was considered at $\mathrm{p}<0.05$.

\section{RESULTS}

One hundred thirty-seven teachers ( 25 males and 112 females) participated in this study. Their ages ranged as follows: 4 (2.9\%) were in their 20s; $14(10.2 \%)$ were in their 30s; 31 (22.6\%) were in their 40s; 76 (55.5\%) were in their 50s; and $12(8.8 \%)$ were in their 60 s (Table 1 ).

The TSES was negatively correlated with harm avoidance $(\mathrm{r}=-0.20, \mathrm{p}=0.017)$ and positively correlated with persistence $(\mathrm{r}=0.35, \mathrm{p}<0.001)$, self-directedness $(\mathrm{r}=0.25, \mathrm{p}=0.004)$, cooperativeness $(r=0.23, p=0.007)$, and self-transcendence $(r=0.21$, $\mathrm{p}=0.013)$. Perceived stress was positively correlated with harm avoidance $(\mathrm{r}=0.41, \mathrm{p}<0.001)$ and negatively correlated with persistence $(\mathrm{r}=-0.18, \mathrm{p}=0.034)$ and self-directedness $(\mathrm{r}=-0.52$, $\mathrm{p}<0.001)$. After Bonferroni correction $[\mathrm{p}<0.007(=0.05 / 7$ TCI subscales)], most correlations remained significant, except for the correlations between the TSES scores and the harm avoidance scores and those between the PSS scores and the persistence scores (Table 2).

To investigate direct and indirect effects of personality variables on perceived stress, the harm avoidance, persistence, and self-directedness scores, which were significantly correlated with the PSS scores, were included in the path model with the TSES and PSS scores. The path analysis showed that harm avoidance directly predicted perceived stress $(\beta=0.37$, $95 \% \mathrm{CI}=0.21-0.53, \mathrm{p}=0.002)$, and self-directedness and persistence predicted one's sense of efficacy $(\beta=0.18,95 \% \mathrm{CI}=$ $0.01-0.39, p=0.05$ and $\beta=0.31,95 \% \mathrm{CI}=0.10-0.47, \mathrm{p}=0.003$ ), which predicted perceived stress $(\beta=-0.21,95 \% \mathrm{CI}=-0.39$ to $-0.02, \mathrm{p}=0.030$ ) (Figure 1).

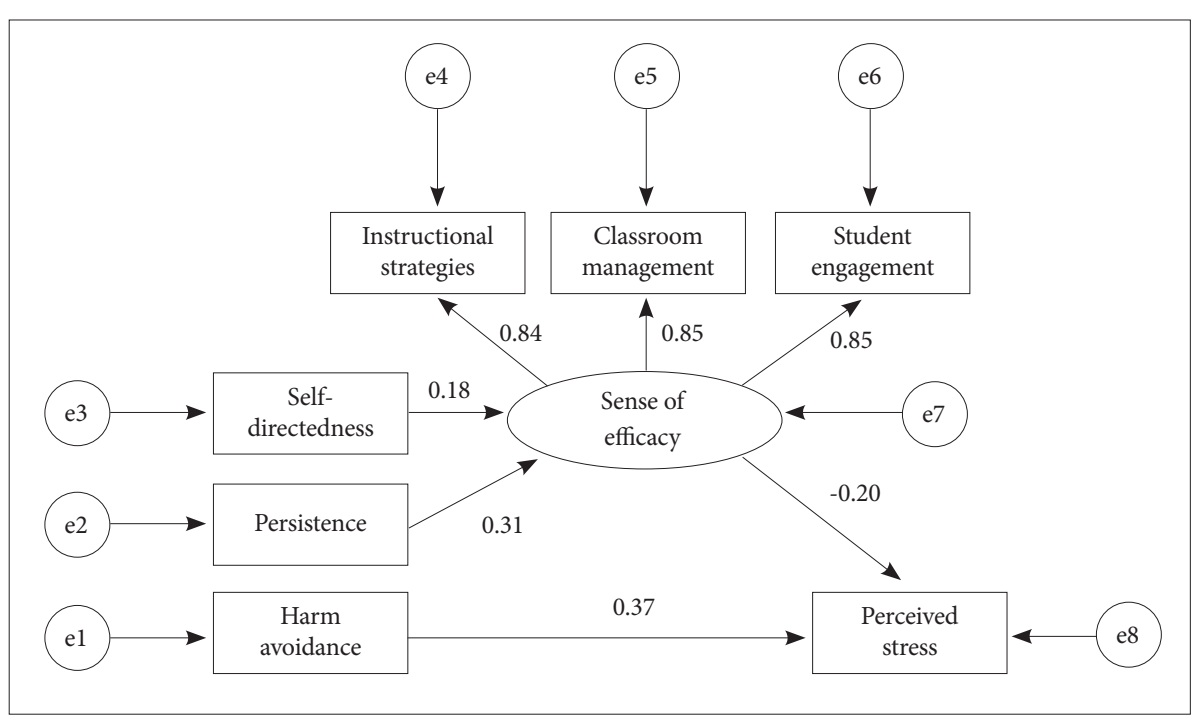

Figure 1. Path analysis showing associations between teachers' personalities, sense of efficacy, and perceived stress. Harm avoidance directly predicts perceived stress. Persistence and self-directedness indirectly predict perceived stress via teachers' sense of efficacy. Only significant paths are presented. E1-E8: residual error terms. 
Table 2. Correlations between teachers' personalities, sense of efficacy and perceived stress

\begin{tabular}{lrrrrr}
\hline & \multicolumn{2}{c}{ Sense of efficacy } & & \multicolumn{2}{c}{ Perceived stress } \\
\cline { 2 - 3 } \cline { 6 - 6 } & \multicolumn{1}{c}{$\mathrm{r}$} & $\mathrm{p}$ & & $\mathrm{r}$ & $\mathrm{p}$ \\
\hline Novelty seeking & 0.02 & 0.808 & & 0.12 & 0.172 \\
Harm avoidance & -0.20 & 0.017 & & 0.41 & $<0.001$ \\
Reward dependence & 0.06 & 0.476 & & 0.01 & 0.952 \\
Persistence & 0.35 & $<0.001$ & & -0.18 & 0.034 \\
Self-directedness & 0.25 & 0.004 & & -0.52 & $<0.001$ \\
Cooperativeness & 0.23 & 0.007 & & -0.16 & 0.063 \\
Self-transcendence & 0.21 & 0.013 & & -0.01 & 0.915 \\
Sense of efficacy & & & & -0.25 & 0.003 \\
\hline
\end{tabular}

\section{DISCUSSION}

The primary goal of this study was to investigate the relationship among teachers' personality factors on the TCI, their sense of efficacy, and their perceived stress. The correlation analysis revealed that two temperament domains (harm avoidance and persistence) and three character domains (self-directedness, cooperativeness, and self-transcendence) were linked with teachers' sense of efficacy. Regarding perceived stress, higher harm avoidance directly predicted higher perceived stress, and lower persistence and self-directedness indirectly predicted higher perceived stress via the mediation of a lower sense of efficacy.

The observed correlations between teachers' temperament/character and their self-efficacy are consistent with previous research by Poulou, ${ }^{26}$ which highlighted the importance of teachers' personalities, capabilities, and motivations as potential sources of teaching efficacy. In this study, teachers' self-efficacy was negatively correlated with harm avoidance, a personality trait characterized by excessive worrying, pessimism, and fearfulness, and positively correlated with persistence (perseverance in spite of frustration), self-directedness (self-determinism/internal locus of control), cooperativeness (agreeableness), and self-transcendence (the experience of spiritual aspects of the self).

The association between specific personality dimensions on the TCI (harm avoidance, persistence, and self-directedness) and perceived stress is comparable with those found in previous studies that used the NEO-PI. In studies using the NEO-PI, personality variables, such as neuroticism, extraversion, and conscientiousness, have been identified as important predictors of stress in teachers. ${ }^{4,5,15}$ Harm avoidance has shown strong positive association with neuroticism and inverse association with extraversion. Persistence has a positive association with conscientiousness. Self-directedness has a strong negative association with neuroticism and a positive association with conscientiousness. ${ }^{27}$ In this respect, the findings from this study are consistent with those from previous studies that considered neuroticism, extraversion, and conscientiousness as potential predictors of teachers' stress. ${ }^{4,5,15}$

Personality variables on the TCI not only had direct effects on perceived stress but also had indirect effects on perceived stress through teachers' sense of efficacy. Such a mediating effect of self-efficacy on the association between the personality and perceived stress was found in a previous population study. ${ }^{28}$ In a population-based study conducted in Denmark, negative associations were found between perceived stress and extroversion, conscientiousness, agreeableness, and openness whilst neuroticism had a positive association, and all associations between Big Five personality dimensions and stress were mediated by general self-efficacy. ${ }^{28}$ Our result was also comparable to the previous research in the Chinese unemployed population, in which self-efficacy partially mediated the relationship between Big Five personality and depressive symptoms. ${ }^{29}$ In the present study, teachers' sense of efficacy was found to mediate the relationship between persistence/selfdirectedness and perceived stress. Teachers who have higher persistence/self-directedness scores may have high self-efficacy, which in turn could lead to lower levels of perceived stress. These findings suggest that interventions that focus on teachers' personalities and self-efficacy might the best approach to reduce teachers' perceived stress and to prevent burnout. It is possible to modify the stress perceptions of teachers who have higher harm avoidance scores by increasing awareness of maladaptive cognition and teachers who have lower persistence and self-directedness scores using by training coping self-efficacy.

The limitations of this study include its cross-sectional study design, which renders it unable to identify causal relationships among an individual's personality, sense of efficacy, and perceived stress. Additionally, factors such as motivation and organizational factors might not be accounted for in this study, and these factors might co-vary with personality and sense of efficacy and be true pathways to stress. Therefore, future prospective studies that consider a variety of covariates are needed to further clarify the causal relationships between personality, self-efficacy, and perceived stress among teachers.

\section{Conclusions}

The results of the present study indicate that harm avoidance might be associated with stress-proneness, while persistence, self-directedness, and sense of efficacy might act as protective resources against stress in Korean teachers. These findings suggest that interventions that focus on both individuals' personalities and self-efficacy may successfully re- 
duce the perceived stress of teachers.

\section{Acknowledgments}

This research was supported by child and adolescent mental health service funded by Seoul National Hospital (3731-311-210-13) and the National Research Foundation of Korea (NRF) Grant funded by the Korean Government (MSIP) (NRF-2014R1A1A3049818).

\section{REFERENCES}

1. Boyle GJ, Borg MG, Falzon JM, Baglioni AJ Jr. A structural model of the dimensions of teacher stress. Br J Educ Psychol 1995;65(Pt 1):4967.

2. Pithers RT. Teacher stress research: problems and progress. Br J Educ Psychol 1995;65(Pt 4):387-392

3. McCormick J, Barnett K. Teachers's attributions for stress and their relationships with burnout. Int J Educl Manage 2011;25:278-293.

4. Kokkinos CM. Job stressors, personality and burnout in primary school teachers. Br J Educ Psychol 2007;77:229-243.

5. Poppla BI, Ilugbo EA. Personality traits as predictors of stress among female teachers in Osun state teaching service. Edo J Counsel 2010;3: 173-188.

6. Tuettemann E, Punch KF. Teachers' psychological distress: the ameliorating effects of control over the work environment. Educ Rev 1992; 44:181-194.

7. Schwarzer R, Hallum S. Perceived teacher self-efficacy as a predictor of job stress and burnout: mediation analyses. Appl Psychol Int Rev 2008;57:152-171.

8. Klassen RM, Tze VMC, Betts SM, Gordon KA. Teacher efficacy research 1998-2008: Sings of progress of unfulfilled promise? Educ Psychol Rev 2011;23:21-43.

9. Vollrath M. Personality and stress. Scand J Psychol 2001;42:335-347.

10. Kaplan HB. Psychosocial Stress from the Perspective of Self Theory. In: Kaplan HB, Editor. Psychosocial Stress: Perspectives on Structure, Theory, Life-Course, and Methods. San Diego, CA: Academic Press; 1996. pp. 175-244.

11. Costa PT, McCrae RR. NEO PI-R Professional Manual. Odessa, FL: Psychological Assessment Resources, Inc; 1992.

12. Cloninger CR. The Temperament and Character Inventory (TCI): A Guide to its Development and Use. St. Louis, MO: Center for Psychobiology of Personality, Washington University; 1994.

13. Besser A, Shackelford TK. Mediation of the effects of the big five personality dimensions on negative mood and confirmed affective expectations by perceived situational stress: a quasi-field study of vacationers. Pers Individ Dif 2007;42:1333-1346.
14. Conard MA, Matthews RA. Modeling the stress process: personality eclipses dysfunctional cognitions and workload in predicting stress. Pers Individ Dif 2008;44:171-181.

15. Grant S, Langan-Fox J. Personality and the occupational stressor-strain relationship: the role of the Big Five. J Occup Health Psychol 2007;12: 20-33.

16. Bandura A. Self-Efficacy: The Exercise of Control. New York: W. H. Freeman; 1997, pp. 2-8.

17. Lisa AR, Ellen LU, John HM. Preliminary investigation of the sources of self-efficacy among teachers of students with autism. Focus Autism Other Dev Disabl 2011;26:67-74.

18. Leganger A, Kraft P. Control constructs: Do they mediate the relation between educational attainment and health behaviour? J Health Psychol 2003;8:361-372.

19. Luszczynska A, Scholz U, Schwarzer R. The general self-efficacy scale: multicultural validation studies. J Psychol 2005;139:439-457.

20. Lazarus RS, Folkman S. Stress, Appraisal, And Coping. New York: Springer; 1984.

21. Sung SM, Kim JH, Yang E, Abrams KY, Lyoo IK. Reliability and validity of the Korean version of the Temperament and Character Inventory. Compr Psychiatry 2002;43:235-243.

22. Tschannen-Morana M, Woolfolk-Hoy A. Teacher efficacy: capturing an elusive construct. Teach Teach Educ 2001;17:783-305.

23. Shin SW, Choi ES. An analysis of structural relationships between adult English educators' instructional leadership, adult English learners' english self-concept, self-leadership, English teacher efficacy and educational satisfaction. Andragogy Today: Int J Adult Cont Educ 2011;14:63-90.

24. Cohen S, Kamarck T, Mermelstein R. A global measure of perceived stress. J Health Soc Behav 1983;24:385-396.

25. Park JO, Seo YS. Validation of the Perceived Stress Scale (PSS) on samples of Korean university students. Korean J Psychol 2010;29:611-629.

26. Poulou M. Personal teaching efficacy and its sources: student teachers' perceptions. Educ Psychol 2007;27:191-218.

27. De Fruyt F, Van De Wiele L, Van Heeringen C. Cloninger's psychobiological model of temperament and character and the five-factor model of personality. Pers Individ Dif 2000;29:441-452.

28. Ebstrup JF, Eplov LF, Pisinger C, Jorgensen T. Association between the five factor personality traits and perceived stress: is the effect mediated by general self-efficacy? Anxiety Stress Coping 2011;24:407-419.

29. Wang Y, Yao L, Liu L, Yang X, Wu H, Wang J, Wang L. The mediating role of self-efficacy in the relationship between Big five personality and depressive symptoms among Chinese unemployed population: a cross-sectional study. BMC Psychiatry 2014;14:61. 